\title{
ANALYTIC CONTINUATION FOR FUNCTIONS OF SEVERAL COMPLEX VARIABLES
}

\author{
BY \\ R. H. CAMERON AND D. A. STORVICK( $\left.{ }^{1}\right)$
}

In our proof of a translation theorem for analytic Feynman integrals [3], a function of two complex variables arose which was analytic in each variable if the other variable was restricted to real values. In this paper we establish some results relating analyticity and separate analyticity for functions of several complex variables.

These results differ from the classical ones [1] in that the functions are not defined initially on complete neighborhoods, only on lower dimensional subsets, and also differ from recent results [2], [4, p. 766] in that our proof gives a precise estimate of the domain of analyticity. This estimate is used in later theorems.

It is convenient to begin with a lemma treating the case of two complex variables in complete detail.

LEMMA 1. Let $f(z, w)$ be defined and bounded on all compact subsets of the three dimensional region obtained by intersecting the product of two circles

$$
\mathscr{R}=\left\{(z, w)|| z\left|<R_{1} \leqq+\infty,\right| w \mid<R_{2} \leqq+\infty\right\}
$$

with the union of two hyperplanes $\mathscr{P}=\{(z, w) \mid(\operatorname{Im} z)(\operatorname{Im} w)=0\}$. For each real $x,-R_{1}<x<R_{1}$, let $f(x, w)$ be analytic in $w$ throughout $|w|<R_{2}$, and for each real $u,-R_{2}<u<R_{2}$, let $f(z, u)$ be analytic throughout $|z|<R$. Then there exists a function $g(z, w)$ and positive numbers $\delta_{1} \geqq R_{1} / 4, \delta_{2} \geqq R_{2} / 4$ such that $g(z, w)$ is analytic in $\mathscr{R}^{*}=\left\{(z, w)|| z\left|<\delta_{1},\right| w \mid<\delta_{2}\right\}$ and $f(z, w) \equiv g(z, w)$ for $(z, w) \in \mathscr{R}^{*} \cap \mathscr{P}$.

Proof. Without loss of generality, assume $R_{1}$ and $R_{2}$ are both finite. Let $F(z, w)=f(a z, b w)$, where $a=R_{1} / 4$ and $b=R_{2} / 4$. The function $F(x, w)$ is analytic in $w$ for $|w|<4$ for each real $x,|x|<4$, and $F(z, u)$ is analytic in $z$ for $|z|<4$ for each real $u,|u|<4$. The function $F(z, w)$ is bounded on all compact subsets of the intersection of $\mathscr{P}$ with $\{(z, w)|| z \mid<4$ and $|w|<4\}$.

Let $B$ be chosen so that $|F(z, w)| \leqq B$ for $(z, w) \in \mathscr{P}$ and $|z| \leqq R^{\prime}$ and $|w| \leqq R^{\prime}$,

Presented to the Society, September 1, 1965; received by the editors August 25, 1965.

(1) Research sponsored by the Air Force Office of Scientific Research, Office of Aerospace Research, United States Air Force, Grant No. AF-AFOSR-381-63. 
where $\left({ }^{2}\right) 5 / 2+\sqrt{ } 2<R^{\prime}<4$. Let us define

$$
F_{m, n}(z, w)=\frac{\partial^{m+n}}{\partial z^{m} \partial w^{n}} F(z, w)
$$

wherever it exists. In particular, these derivatives exist for the above region when $m=0$ if $z$ is real and when $n=0$ if $w$ is real.

By Cauchy's inequality we have

$$
\left|F_{m, 0}(z, w)\right| \leqq \frac{m ! B}{\left(R^{\prime}-1\right)^{m}} \quad \text { if }|z| \leqq 1, \quad \operatorname{Im} w=0 \quad \text { and } \quad|\operatorname{Re}(w)| \leqq R^{\prime},
$$

and

$$
\left|F_{0, n}(z, w)\right| \leqq \frac{n ! B}{\left(R^{\prime}-1\right)^{n}} \quad \text { if }|w| \leqq 1, \quad \operatorname{Im} z=0 \text { and }|\operatorname{Re}(z)| \leqq R^{\prime} .
$$

For real $(x, u)$ we expand $F(x, u)$ in a double Legendre series, thus

$$
F(x, u) \sim \sum_{m, n=0}^{\infty} a_{m, n} P_{m}(x) P_{n}(u)
$$

and we have

$$
a_{m, n}=\left(m+\frac{1}{2}\right)\left(n+\frac{1}{2}\right) \int_{-1}^{+1} \int_{-1}^{+1} F(x, u) P_{m}(x) P_{n}(u) d x d u
$$

By Rodriques' formula and integration by parts

$$
\begin{aligned}
a_{m, n} & =\left(m+\frac{1}{2}\right) \frac{(n+1 / 2)}{2^{n} n !} \int_{-1}^{+1} \int_{-1}^{+1} F(x, u) P_{m}(x) \frac{d^{n}}{d u^{n}}\left(u^{2}-1\right)^{n} d u d x \\
& =\frac{(m+1 / 2)(n+1 / 2)}{(-1)^{n} 2^{n} n !} \int_{-1}^{+1} \int_{-1}^{+1} P_{m}(x) F_{0, n}(x, u)\left(u^{2}-1\right)^{n} d u d x
\end{aligned}
$$

We therefore obtain

$$
\left|a_{m, n}\right| \leqq \frac{4(m+1 / 2)(n+1 / 2) B}{2^{n}\left(R^{\prime}-1\right)^{n}}
$$

and similarly

$$
\left|a_{m, n}\right| \leqq \frac{4(m+1 / 2)(n+1 / 2) B}{2^{m}\left(R^{\prime}-1\right)^{m}} .
$$

$\left.{ }^{2}\right)$ The number 4 which occurs in the statement of the theorem and correspondingly in the proof is used because it is a convenient number which is slightly greater than $5 / 2+\sqrt{ } 2$. As we shall see, the latter number is important in obtaining the convergence of the double Legendre series which will be basic to this proof. 
Now $m$ and $n$ cannot both be less than $q=(m+n) / 2$. If $m \geqq q$, we have

$$
\left|a_{m, n}\right| \leqq \frac{4(m+1 / 2)(n+1 / 2) B}{2^{m}\left(R^{\prime}-1\right)^{m}} \leqq \frac{(2 m+1)(2 n+1) B}{2^{q}\left(R^{\prime}-1\right)^{q}}
$$

Similarly, if $n \geqq q$ we obtain

$$
\left|a_{m, n}\right| \leqq \frac{(2 m+1)(2 n+1) B}{2^{q}\left(R^{\prime}-1\right)^{q}} .
$$

Thus, in either case

$$
\left|a_{m, n}\right| \leqq \frac{(2 m+1)(2 n+1) B}{\left[2\left(R^{\prime}-1\right)\right]^{(m+n) / 2}}
$$

By Laplace's integral formula:

$$
P_{n}(z)=\frac{1}{\pi} \int_{0}^{\pi}\left\{z+\left(z^{2}-1\right)^{1 / 2} \cos \varphi\right\}^{n} d \varphi,
$$

we have for $|z| \leqq 1$ that $\left|z+\left(z^{2}-1\right)^{1 / 2} \cos \varphi\right|<1+\sqrt{ } 2$ so that $\left|P_{m}(z)\right| \leqq(1+\sqrt{ } 2)^{m}$ if $|z| \leqq 1$. Thus for $|z| \leqq 1$ and $|w| \leqq 1$,

$$
\left|a_{m, n} P_{m}(z) P_{n}(w)\right| \leqq \frac{(2 m+1)(2 n+1) B}{\left[2\left(R^{\prime}-1\right)\right]^{(m+n) / 2}}(1+\sqrt{ } 2)^{(m+n)}
$$

But since $R^{\prime}>5 / 2+\sqrt{ } 2$, we have $2\left(R^{\prime}-1\right)>(1+\sqrt{ } 2)^{2}$, and since

$$
\left|a_{m, n} P_{m}(z) P_{n}(w)\right| \leqq(2 m+1)(2 n+1) B\left(\frac{1+\sqrt{ } 2}{\sqrt{ }\left(2\left(R^{\prime}-1\right)\right)}\right)^{m+n}
$$

the double series $\sum_{m, n=0}^{\infty} a_{m, n} P_{m}(z) P_{n}(w)$ converges absolutely and uniformly for $|z|<1,|w|<1$. It converges to a function $g(z, w)$ which is analytic in $(z, w)$ in the four dimensional region $|z|<1,|w|<1$. Therefore the original function $f(z, w)$ has an analytic continuation to $\mathscr{R}^{*}=\left\{(z, w)|| z\left|<a=R_{1} / 4,\right| w \mid<b=R_{2} / 4\right\}$. This completes the proof of the lemma.

Note. It is clear that we may modify the hypothesis so that the centers of the two circles are translated to any two points and the diameters are rotated into any diameters.

An example as simple as $f(z, w)=1 /(z+w-i)$ shows that the $\delta=1 / 4$ obtained above cannot equal one. However, the fact that we have a precise estimate, a lower bound on $\delta$, enables us to considerably extend the domain of analyticity in the case where $f(z, w)$ is an entire function in one variable.

Let us now assume that in the lemma $R_{1}=1, R_{2}=\infty$, and let us restrict our attention to the $z$-plane. By the lemma, there exists a disk $|z|<\delta=1 / 4$ contained in 
the domain of analyticity $\left({ }^{3}\right)$. By a simple translation and change of scale, every disk $\left(x-x_{0}\right)^{2}+y^{2}<\delta\left(1-\left|x_{0}\right|\right)^{2}$ is contained in the domain of analyticity. Hence the diamond shaped region $D$ containing the origin $z=0$ and bounded by the lines

$$
\frac{x}{1}+\frac{y}{\delta}=1, \quad \frac{x}{-1}+\frac{y}{-\delta}=1, \quad \frac{x}{1}+\frac{y}{-\delta}=1, \quad \text { and } \frac{x}{-1}+\frac{y}{\delta}=1
$$

is contained in the domain of analyticity.

As before, we obtain an obvious generalization by change of scale, translation, and rotation.

Terminology. In the future, the term "diamond shaped region" or "diamond" will mean a region similar to the one described above, bounded by a rhombus with one diagonal exactly one-fourth the length of the other.

Let us now consider repeated application of the above procedure, i.e., take any line segment $l$ which is contained in $D$ and which is the diameter of a circle contained in $|z|<1$ and construct the diamond shaped region $D_{l}$ on $l$. We now repeat using $D \cup D_{l}$, etc. If we consider all possible star shaped regions centered at the origin to which $f$ can be extended analytically $\left({ }^{4}\right)$, the union of all such star shaped regions $R=\bigcup_{\alpha} D_{\alpha}$ is again star shaped with respect to this origin and as we shall now prove, convex.

If $R$ were not convex, there would exist two points $P, Q \in R$ with the line segment $\overline{P Q} \notin R$. There exists a line segment $\overline{p q}$ parallel to $\overline{P Q}$, with end points on the lines from the origin to $P$ and $Q$, which contains a point $S, S \notin R$ such that the line segment is closest to the origin. We now construct a diamond on a line segment $\overline{r s} \subset R, \overline{r s}$ parallel to $\overline{p q}$, with ends on $\overline{O P}$ and $\overline{O Q}$, and closer to the origin. If $\overline{r s}$ is sufficiently close to $\overline{p q}$, the diamond shaped region constructed on $\overline{r s}$ contains $S$ and therefore contradicts the assumption that $S \notin R=\bigcup_{\alpha} D_{\alpha}$. Thus the region $R$ is convex.

Since $R$ is a convex set, $\Gamma=F_{r}(R)$ is a rectifiable curve and possesses a tangent except, at most, at a countable set of points. If $R$ is not the unit disk $|z|<1$, there exists a point $p,|p|<1, p \in \Gamma$ such that at $p, \Gamma: z=z(t)$ has a tangent. Let $T$ be the tangent line to $\Gamma$ at $p=z\left(t_{0}\right)$. Then there exists a $\delta_{0}>0$ such that for all $t,\left|t-t_{0}\right|<\delta_{0}$ the secant line $S_{t}$ joining $z(t)$ to $z\left(t_{0}\right)$ makes an angle $\alpha(t)$ with $T$ of size $<\arctan (1 / 4)$. We form the circle $C_{p}:|z-p|=1-|p|$ and pick an integer $n>0$ such that $1 / n<\delta_{0}$ and such that $z\left(t_{0} \mp 1 / n\right)=q_{n}$ lies in the interior of $C_{p}$. The diamond constructed on $\overline{p q}_{n}$ contains points exterior to $R$ since the angle of the diamond is $>\arctan (1 / 4)$. This contradicts the definition of $R$ as the domain $R=\bigcup_{\alpha} D_{\alpha}$; therefore $R$ is the unit disk, $R=\{|z|<1\}$.

$\left({ }^{3}\right)$ I.e., the domain in the $z$-plane such that the function can be extended so as to be analytic in $(z, w)$ for all $z$ in the particular domain and all complex $w$.

( ${ }^{4}$ See the previous footnote. 
We now formalize the results of the foregoing observations in our second lemma.

Lemma 2. If $f(z, w)$ satisfies the hypothesis of Lemma 1 and if $R_{2}=\infty$, then the conclusion of Lemma 1 holds with $\delta_{1}=R_{1}$ and $\delta_{2}=\infty$.

The techniques above are not restricted in applicability to the case of two complex variables. Indeed, the proof of the lemma can be immediately extended to yield the following theorem.

THEOREM 1. Let $\Omega_{j}$ denote a disk about the origin of radius $R_{j}$ and real diameter $L_{j}$ for $j=1,2, \ldots, n$. Let $f\left(z_{1}, z_{2}, \ldots, z_{n}\right)$ be defined and bounded on all compact subsets of

$S=\left(\Omega_{1} \times L_{2} \times \cdots \times L_{n}\right) \cup\left(L_{1} \times \Omega_{2} \times L_{3} \times \cdots \times L_{n}\right) \cup \cdots \cup\left(L_{1} \times \cdots \times L_{n-1} \times \Omega_{n}\right)$.

Let $f\left(z_{1}, \ldots, z_{n}\right)$ be analytic for $z_{1} \in \Omega_{1}$ for $\left(z_{2}, \ldots, z_{n}\right) \in L_{2} \times \cdots \times L_{n}$, be analytic for $z_{2} \in \Omega_{2}$ for $\left(z_{1}, z_{3}, \ldots, z_{n}\right) \in L_{1} \times L_{3} \times \cdots \times L_{n}, \ldots$, be analytic for $z_{n} \in \Omega_{n}$ for $\left(z_{1}, z_{2}, \ldots, z_{n-1}\right) \in L_{1} \times L_{2} \times \cdots \times L_{n-1}$. Then there exists $\delta_{1}, \delta_{2}, \ldots, \delta_{n}>0$ and $a$ function $g\left(z_{1}, z_{2}, \ldots, z_{n}\right)$ analytic in $\Delta=\Delta_{1} \times \Delta_{2} \times \cdots \times \Delta_{n}$, where $\Delta_{j}$ is a disk of radius $\delta_{j} \geqq(2 / 5)^{n-2} R_{j} / 4$ about the origin in the $z_{j}$ plane for $j=1,2, \ldots, n$ and $f\left(z_{1}, \ldots, z_{n}\right) \equiv g\left(z_{1}, z_{2}, \ldots, z_{n}\right)$ on $S \cap \Delta$.

Proof. The proof is the obvious analogue of that of Lemma 1, with the numbers $(1+\sqrt{ } 2)^{n} / 2+1$ and $4(5 / 2)^{n-2}$ taking the places of $5 / 2+\sqrt{ } 2$ and 4 respectively. It is easy to see that the first of these numbers is less than the second when $n>1$, while the theorem holds trivially when $n=1$. Thus the theorem holds in general.

An application of the principle of analytic continuation and the monodromy theorem for several complex variables yields the following theorem.

THEOREM 2. Let $\Omega_{1}$ be a simply connected domain in the $z_{1}$-plane and let $\Omega_{j}$ denote the $z_{j}$-plane, $\left|z_{j}\right|<\infty$ for $j=2,3, \ldots, n$. Let $L_{1}$ be a line segment, $L_{1} \subset \Omega_{1}$ and let $L_{j}$ denote a complete line, $L_{j} \subset \Omega_{j}$ for $j=2, \ldots, n$. Let $f\left(z_{1}, \ldots, z_{n}\right)$ be defined and bounded on all compact subsets of

$S=\left(\Omega_{1} \times L_{2} \times \cdots \times L_{n}\right) \cup\left(L_{1} \times \Omega_{2} \times L_{3} \times \cdots \times L_{n}\right) \cup \cdots \cup\left(L_{1} \times \cdots \times L_{n-1} \times \Omega_{n}\right)$.

Let $f\left(z_{1}, \ldots, z_{n}\right)$ be analytic in $\Omega_{1}$ for $\left(z_{2}, \ldots, z_{n}\right) \in L_{2} \times \cdots \times L_{n}$, and let $f\left(z_{1}, \ldots, z_{n}\right)$ be analytic in $\Omega_{2}$ for $\left(z_{1}, z_{3}, \ldots, z_{n}\right) \in L_{1} \times L_{2} \times \cdots \times L_{n}$, and $\ldots$, let $f\left(z_{1}, \ldots, z_{n}\right)$ be analytic in $\Omega_{n}$ for $\left(z_{1}, \ldots, z_{n-1}\right) \in L_{1} \times \cdots \times L_{n-1}$. Then there exists a function $g\left(z_{1}, \ldots, z_{n}\right)$ which is analytic in $\Omega_{1} \times \cdots \times \Omega_{n}$ such that $g\left(z_{1}, \ldots, z_{n}\right) \equiv f\left(z_{1}, \ldots, z_{n}\right)$ on $S$. 
Proof. For the case in which $\Omega_{1}$ is a disk and $L_{1}$ a diameter of $\Omega_{1}$, the proof is the obvious $n$-dimensional extension of the proof of Lemma 2 . For the case in which $\Omega_{1}$ is a disk and $L_{1}$ is a segment of a diameter and is concentric with $\Omega_{1}$, essentially the same argument works. Now for the general case, consider any point $P$ in $\Omega_{1}$ and connect it to the common center $Q_{1}$ of $\Omega_{1}$ and $L_{1}$ by a smooth curve $C$ in $\Omega_{1}$. Apply the preceding case to extend analytically to the largest disk about $Q_{1}$ that lies in $\Omega_{1}$. Repeat the process for a point $Q_{2}$ that lies on the intersection of $C$ with this disk. The usual argument shows that $P$ can be reached by a finite set of repetitions of this process, and the monodromy theorem shows that a single valued extension can be made to the whole of $\Omega_{1}$. Thus the theorem is proved.

\section{BIBLIOGRAPHY}

1. S. Bochner and W. T. Martin, Several complex variables, Princeton Univ. Press, Princeton, N. J., 1948.

2. F. Browder, Real analytic functions on product spaces and separate analyticity, Canad. J. Math. 13 (1961), 650-656.

3. R. H. Cameron and D. A. Storvick, A translation theorem for analytic Feynman integrals, Trans. Amer. Math. Soc. 125 (1966), 1-6.

4. R. A. Kunze and E. M. Stein, Uniformly bounded representations. II, Amer. J. Math. 83 (1961), 723-786.

UNIVERSITY OF MINNESOTA, MinNEAPOLIS, MinNESOTA 